\title{
Oral and dermal absorption of chlorpyrifos: a human volunteer study
}

\author{
P Griffin, H Mason, K Heywood, J Cocker
}

\begin{abstract}
Objectives-To determine the kinetics of elimination of urinary dialkylphosphate metabolites after oral and dermally applied doses of the organophosphate pesticide chlorpyrifos to human volunteers and to determine whether these doses affected plasma and erythrocyte cholinesterase activity.
\end{abstract}

Method-Five volunteers ingested $1 \mathrm{mg}$ (2852 nmol) of chlorpyrifos. Blood samples were taken over 24 hours and total void volumes of urine were collected over 100 hours. Four weeks later $28.59 \mathrm{mg}$ (81567 nmol) of chlorpyrifos was administered dermally to each volunteer for 8 hours. Unabsorbed chlorpyrifos was washed from the skin and retained for subsequent measurement. The same blood and urine sampling regime was followed as for the oral administration. Plasma and erythrocyte cholinesterase concentrations were determined for each blood sample. The concentration of two urinary metabolites of chlorpyrifos-diethylphosphate and diethylthiophosphate-was determined for each urine sample.

Results-The apparent elimination half life of urinary dialkylphosphates after the oral dose was 15.5 hours and after the dermal dose it was 30 hours. Most of the oral dose (mean (range) 93\% (55-115\%)) and $1 \%$ of the applied dermal dose was recovered as urinary metabolites. About half $(53 \%)$ of the dermal dose was recovered from the skin surface. The absorption rate through the skin, as measured by urinary metabolites was $456 \mathrm{ng} / \mathrm{cm}^{2} / \mathrm{h}$. Blood plasma and erythrocyte cholinesterase activity did not fall significantly during either dosing regime.

Conclusion-An oral dose of chlorpyrifos was readily absorbed through the skin and almost all of the dose was recovered as urinary dialkylphosphate metabolites. Excretion was delayed compared with the oral dose. Only a small proportion of the applied dose was recovered during the course of the experiment. The best time to collect urine samples for biological monitoring after dermal exposure is before the shift the next day. The amounts of chlorpyrifos used did not depress acetyl cholinesterase activity but could be readily detected as urinary dialkylphosphate metabolites indicating that the urinary assay is a more sensitive indicator of exposure.

(Occup Environ Med 1999;56:10-13)

Keywords: biological monitoring; chlorpyrifos; organophosphorus pesticide
The organophosphate pesticide chlorpyrifos (O,O-diethyl-O-(3,5,6-trichloro-2-pyridyl) phosphothioate) is a non-systemic cholinesterase inhibitor used to combat agricultural and horticultural insect pests. Several pesticide formulations containing chlorpyrifos have been marketed by different manufacturers. These include Dursban, Lorsban, Brodan, Eradex, and Spannit. A study of the metabolism of chlorpyrifos in rats has shown that the major metabolites are diethylphosphate, diethylthiophosphate, and 3,5,6-trichloro-2-pyridinol. ${ }^{1}$

Chlorpyrifos is widely used as a replacement for chlorinated hydrocarbon and some other less effective organophosphate pesticides ${ }^{2}$ and there is, therefore, increasing potential for occupational exposure of humans. Due to its lipophilic nature chlorpyrifos, along with many organophosphorous pesticides, is likely to be readily absorbed through the skin. A previous publication has shown a close correlation between dermal exposure and excreted metabolites ${ }^{3}$ and the United Kingdom's Health and Safety Executive publication EH40/98 gives chlorpyrifos a skin notation. This suggests that biological monitoring might be the appropriate method for assessing uptake of chlorpyrifos, whether from dermal or mixed exposure. Biological monitoring can be carried out by measuring the inhibition of plasma and erythrocyte cholinesterase. ${ }^{5}$ A different method which can be used for biological monitoring is the measurement of urinary metabolites of organophosphorus pesticides. Some knowledge of the kinetics of metabolite excretion is necessary to know the optimum time for urine collection. Also it is possible to determine how much of a given dose is excreted as metabolites. The pharmacokinetics of chlorpyrifos absorption and excretion, as measured by the excretion of 3,5,6-trichloro-2-pyridinol and inhibition of cholinesterase, has been determined. ${ }^{6}$ However, measurement of dialkylphosphate metabolites is increasingly being used to determine exposure to organophosphorus pesticides. ${ }^{78}$ These tests can be used to measure exposure to a range of pesticides and therefore have a greater applicability to biological monitoring than measurement of metabolites that are specific to one organophosphate pesticide. However, the dialkylphosphate metabolites of chlorpyrifos may have different excretion rates from 3,5,6trichloro-2-pyridinol.

The aim of this work was to determine the elimination kinetics of a known oral and dermal dose of chlorpyrifos as measured by urinary dialkylphosphate metabolites. 


\section{Method}

POPULATION AND SAMPLE COLLECTION

Approval for this study was obtained from the Health and Safety Executive research ethics committee (ETHCOM/REG/95/12). Five volunteers, four men and one women, age range: 26-45, weight range: $73-92 \mathrm{~kg}$ ingested $1 \mathrm{mg}$ $(2852 \mathrm{nmol})$ of analytical grade chlorpyrifos (Promochem) applied to a sugar cube. This dose is half that which would be absorbed if a subject were exposed to the Health and Safety Executive occupational exposure standard of $0.2 \mathrm{mg} / \mathrm{m}^{3}$ over an 8 hour period, assuming that the subject inhaled $10 \mathrm{~m}^{3}$ of air over this period and there was complete absorption. Blood samples were collected through an indwelling catheter (Veneflon) from a vein in the forearm. A sample of blood and urine was taken from each volunteer before exposure. Thereafter, samples of blood and total void volumes of urine were collected every 2 hours after administration for 8 hours. A blood sample was taken next day. From day 2 to day 5 total void volumes of urine were collected for 4 hour periods during the day, and for an 8 hour period overnight. All samples were marked with date and time of collection and the volume of urine collected was recorded.

Four weeks after the oral dose, $28.59 \mathrm{mg}$ (81 $567 \mathrm{nmol}$ ) of chlorpyrifos was administered to the skin of the same volunteers by spreading $100 \mu \mathrm{l}$ of a commercial preparation of chlorpyrifos (Durban 4, Dow Elanco), diluted in water, on to an area of $78 \mathrm{~cm}^{2}$ of the inner forearm. Nolan et $a l^{6}$ estimated that about $1.35 \%$ of a dermal dose was absorbed. From this figure we estimated that the amount absorbed from our dermal dose would not exceed that absorbed from our oral dose. The forearm area was covered with a raised impermeable plastic container which was left in place for 8 hours; at the end of this period the inside of the container was swabbed out and the skin washed with water and soap solution and three cotton wool swabs.

The cotton wool swabs were extracted with $20 \mathrm{ml}$ ethyl acetate on a tube roller for $30 \mathrm{~min}$ utes. The liquid was decanted into screw capped tubes and the swabs compressed in a syringe barrel. The liquid was expressed into fresh tubes. These tubes were then put on a rotator for 30 minutes, centrifuged at $700 \mathrm{~g}$ for 20 minutes, and the organic layer decanted to a fresh tube. The organic layer was evaporated to dryness under nitrogen and the residue dissolved in $2 \mathrm{ml}$ acetonitrile.

Samples of blood and urine were taken with the same protocol as used for oral administration. Blood samples were collected from the contralateral arm.

ANALYSIS OF CHLORPYRIFOS AND METABOLITES Urinary dialkyl phosphate metabolites were measured by an established method. ${ }^{7}$ The concentration of the diethyl phosphate and diethyl thiophosphate metabolites of chlorpyrifos were summed for each measurement and the results calculated as concentration per volume of urine and as values corrected for creatinine.
Table 1 Total amount of urinary dialkyl phosphate metabolites recovered from volunteers after an oral dose of chlorpyrifos

\begin{tabular}{clc}
\hline Volunteer & $\begin{array}{l}\text { Total recovered as urinary } \\
\text { metabolites (nmol) }\end{array}$ & $\%$ Recovered \\
\hline 1 & 3264 & 115 \\
2 & 2460 & 86 \\
3 & 2668 & 94 \\
4 & 2045 & 72 \\
5 & 1567 & 55 \\
Mean & 2401 & 93 \\
\hline
\end{tabular}

Chlorpyrifos was measured with high performance liquid chromatography (HPLC) with ultraviolet (UV) detection at $290 \mathrm{~nm}$. A standard curve was prepared over the range 0-30 $\mathrm{mg} / \mathrm{l}$. Injections of samples or standards $(2 \mu \mathrm{l})$ were made on to an ODS 2 column (Phenomenex) and eluted with a gradient from $100 \%$ water to $100 \%$ acetonitrile over 15 minutes. The coefficient of variation for this assay was $11 \%$.

ANALYSIS OF CHOLINESTERASE ACTIVITY

Plasma and erythrocyte cholinesterase values were measured, within 24 hours, by the method of Mason and Lewis. ${ }^{9}$

SOFTWARE

Data manipulation was carried out on an excel spreadsheet (Microsoft) and analysis of excretion results, to obtain elimination half lives, was carried out with Prism software (Graphpad Software).

\section{Results}

ORAL EXPOSURE

Each volunteer consumed $2852 \mathrm{nmol}$ chlorpyrifos. Only one (volunteer 1, day 1, 8-10 hour) of the 100 urine sample points were missed. The total amounts of dialkyl phosphate (diethyl and diethylthio) metabolites recovered in urine for each volunteer are shown in table 1.

Chlorpyrifos metabolite excretion expressed as values corrected for creatinine is shown in figure 1, and as total ethyl metabolites excreted per hour in figure 2. Chlorpyrifos was rapidly absorbed, metabolised, and eliminated as shown by the rise to maximum levels of excretion at 7 hours. This was followed by an exponential excretion profile. The apparent half times of elimination were calculated from values corrected for creatinine (15.5 hours) and total dialkylphosphate metabolites (15.8

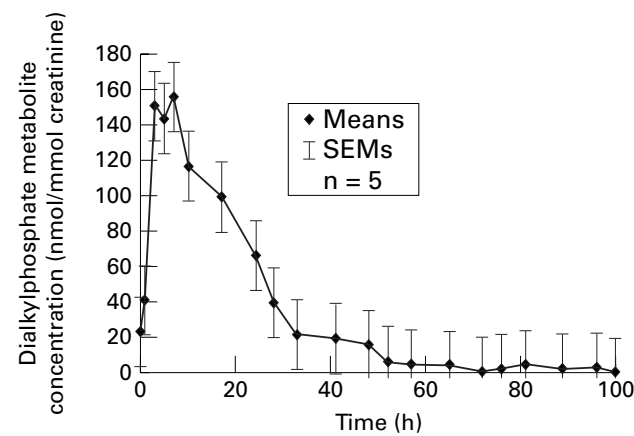

Figure 1 Time course of dialkylphosphate excretion corrected for urinary creatinine concentration for the oral dose. 


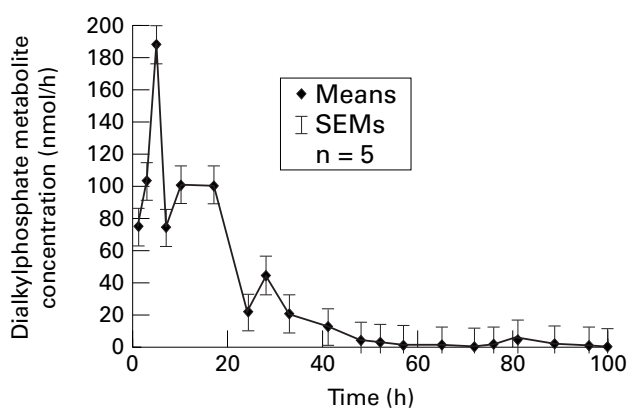

Figure 2 Time course of dialkylphosphate excretion after the oral dose expressed as total ethyl metabolites excreted over time.

hours). Although both sets of data gave similar half lives the values corrected for creatinine fitted the one compartment exponential equation (95\% confidence interval $(95 \% \mathrm{CI})$ of the half life 12.5 to $\left.20.5 \mathrm{~h}, R^{2}=0.85\right)$ better than the data derived from total metabolite excretion (95\% CI for the half life 10.39 to $32.71 \mathrm{~h}$, $\left.R^{2}=0.55\right)$. For this reason we used values corrected for creatinine to calculate apparent half times of elimination and the time of maximum concentration of metabolites in urine.

\section{DERMAL EXPOSURE}

Each volunteer was exposed to a dose of 81567 $\mathrm{nmol}$ in $100 \mu \mathrm{l}$ of the concentrate-water formulation. This was spread over $78 \mathrm{~cm}^{2}$ of skin on the lower forearm. This is an exposure of $1046 \mathrm{nmol} / \mathrm{cm}^{2}$ of skin. A graph of excretion of dialkyl phosphate metabolites as measured by values corrected for creatinine is shown in figure 3 and as total ethyl metabolites per hour in figure 4 . The fraction of the total dose recovered in the urine as dialkylphosphate metabolites over the 5 day sampling period was $1 \%(809 \mathrm{nmol})$, and 53\% (42925 $\mathrm{nmol})$ was recovered from skin washings. If it is assumed

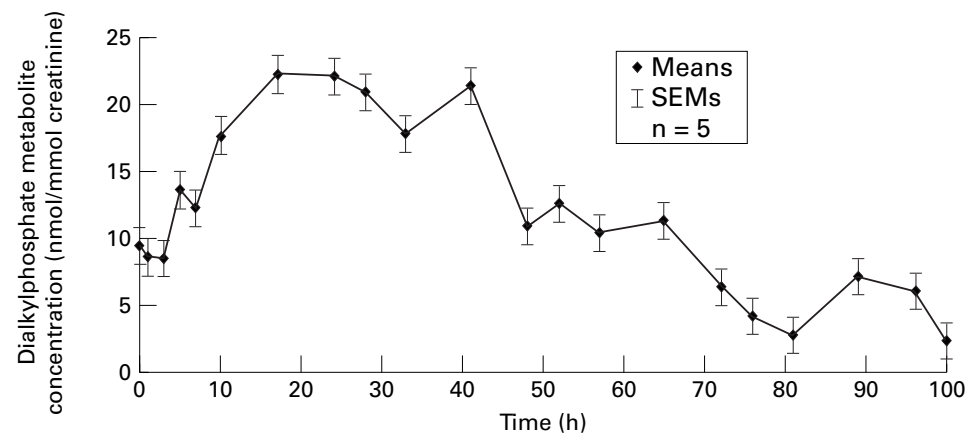

Figure 3 Time course of dialkylphosphate excretion corrected for urinary creatinine concentration for the dermal dose.

Table 2 Total amount of urinary dialkylphosphate metabolites and chlorpyrifos recovered from volunteers after a dermal dose of chlorpyrifos

\begin{tabular}{llll}
\hline Volunteer & $\begin{array}{l}\text { Total recovered as } \\
\text { urinary metabolites } \\
\text { (nmol) }\end{array}$ & $\begin{array}{l}\text { Total recovered from } \\
\text { skin washings (nmol) }\end{array}$ & $\begin{array}{l}\text { Total recovered } \\
\text { (nmol) }\end{array}$ \\
\hline 1 & $986(1.2)$ & not available & \\
2 & $581(0.7)$ & $46734(57.2)$ & $47315(58.6)$ \\
3 & $1264(1.5)$ & $43378(53.2)$ & $44642(54.7)$ \\
4 & $765(0.9)$ & $52156(63.9)$ & $52921(64.8)$ \\
5 & $449(0.6)$ & $29435(36.0)$ & $29884(36.6)$ \\
Mean & $809(1.0)$ & $42925(52.6)$ & $43734(53.6)$ \\
\hline
\end{tabular}

Percentages are in parentheses..

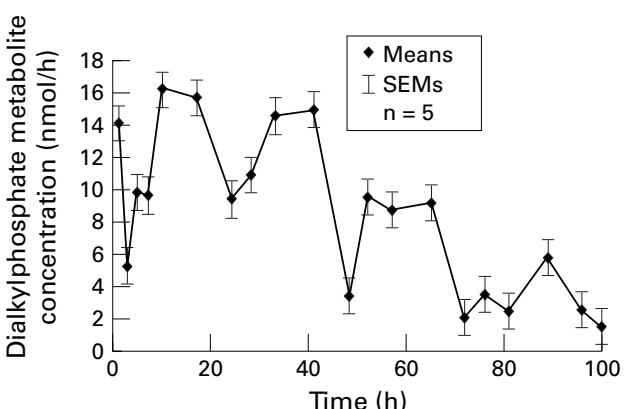

Figure 4 Time course of dialkylphosphate excretion after the dermal dose expressed as total ethyl metabolites excreted over time.

that all diethyl metabolites were derived from the dose of chlorpyrifos and that absorption was substantially reduced or eliminated after washing at 8 hours after application then this $809 \mathrm{nmol}$ represents an absorption rate of 1.30 $\mathrm{nmol}$ of chlorpyrifos $/ \mathrm{cm}^{2}$ skin/h or $456 \mathrm{ng} /$ $\mathrm{cm}^{2} / \mathrm{h}$. The total recovery for each volunteer is shown in table 2 . The maximum concentration of metabolites in urine as measured in $\mathrm{nmol} / \mathrm{mmol}$ creatinine occurred 24 hours after the start of the experiment and 9-16 hours after the dose had been washed off the skin.

The maximum rate of excretion was approximately $16 \mathrm{nmol} /$ hour after a dermal dose of $81567 \mathrm{nmol}$ compared with 10 times this value for the oral dose of $2852 \mathrm{nmol}$. Figure 3 shows the excretion profile of the dermal dose when the results of dialkyl phosphate metabolites are presented as $\mathrm{nmol} / \mathrm{mmol}$ creatinine.

The apparent elimination half life of the urinary dialkyl phosphate metabolites for the dermal dose as measured by results corrected for creatinine was 30 hours after the start of the experiment $\left(95 \%\right.$ CI $\left.25-39 \quad \mathrm{~h}, R^{2}=0.62\right)$ assuming a final urinary dialkylphosphate concentration of zero. This assumption is based on the data for the oral exposure in which the final results fell below the detection limit.

The apparent elimination half life of the urinary dialkyl phosphate metabolites for the dermal dose as measured by the rate of excretion expressed as nmol/hour was 41 hours after the start of the experiment (95\% CI 27-88 h, $R^{2}=0.22$ ) assuming a final urinary dialkylphosphate concentration of zero. Reference to figure 4 shows that the curve shows dips 24,48 , and 72 hours after the start of the experiment.

The total amount of metabolites excreted after the oral dose varied. An average of $93 \%$ of the oral dose was excreted as dialkylphosphate metabolites. After the dermal dose $1 \%$ was excreted as dialkylphosphate metabolites and $53 \%$ was recovered as chlorpyrifos from skin washings. This means that up to $46 \%$ of the dermal dose has not been accounted for as metabolites in urine or as chlorpyrifos in skin washings.

\section{BLOOD PLASMA AND ERYTHROCYTE}

CHOLINESTERASE INHIBITION

Blood plasma and erythrocyte cholinesterase activity did not fall below $90 \%$ of that before exposure during either dosing regime, except for one result for one volunteer during the dermal administration. This occurred after the dermal 
dose and the fall to $86 \%$ did not fit with the trend shown by the other results from this subject.

\section{Discussion}

The dermal application had an apparent half life nearly twice as long as the oral dose both for the absorption related phase of the time course as well as the elimination phase. The dermal dose did not fit an exponential elimination profile as well as the oral dose $\left(R^{2}=0.62\right.$ for the dermal dose compared with $R^{2}=0.85$ for the oral dose). The difference in the apparent elimination profile may be due to continued absorption, storage, and metabolism in the skin and associated tissues. After the oral dose we measured almost complete recovery of the dose as urinary metabolites. After the dermal dose we only recovered $1 \%$ as metabolites and only $53 \%$ as skin washings. In a complimentary study we exposed skin to chlorpyrifos in vitro with a similar protocol to the dermal absorption study reported here. In the in vitro studies we obtained about $90 \%$ recovery suggesting that recovery from skin swabs was complete (unpublished data).

We found that after a dermal dose the maximum concentration of urinary dialkylphosphates was reached after 17-24 hours, and the half life was 30 hours. After an oral dose the half life was 15.5 hours. The half life for elimination, after both an oral and dermally applied dose of chlorpyrifos, as measured by urinary 3,5,6-trichloro-2-pyridinol, has been quoted as 26.9 hours. $^{6}$ The differences between the results may be due to variation in the elimination of the different metabolites measured by Nolan $e t a l^{6}$ and ourselves. In another study of workers occupationally exposed to chlorpyrifos the maximum concentration of urinary 3,5,6trichloro-2-pyridinol has been quoted as being reached after $18-24$ hours. ${ }^{3}$ This is in agreement with our findings.

Nolan et $a l^{6}$ reported that $70 \%$ of an oral dose and $1.3 \%$ of a dermally applied dose were excreted as urinary metabolites, which compares favourably with our findings of recovery of $93 \%$ of an oral dose and $1 \%$ of a dermal dose as urinary metabolites. There was a difference in the way that the dermally applied dose was applied between our study and the study of Nolan et al. ${ }^{6}$ Nolan et al applied chlorpyrifos dissolved in dipropylene glycol methyl ether with a non-occluded dressing, ${ }^{6}$ whereas we used a chlorpyrifos suspension in water as a vehicle and we used an occluded dressing. In our study the vehicle used for applying the chlorpyrifos was chosen to mimic, as closely as possible, occupational exposure for workers handling this product.

In the field total void urine collections are impractical and results for dialkylphosphates are often expressed as values corrected for creatinine. We determined our kinetic data from values corrected for creatinine. There was less variability in these values than in the total amounts of dialkylphosphate metabolites excreted. This is illustrated in figures 3 and 4 where the values corrected for creatinine show a smoother curve. The falls in the rate of excretion at 24,48 , and 72 hours (fig 4) may have been due to the relatively short sampling periods during the day leading to a magnification of the effect of incomplete urine collections by volunteers. A good correlation between data on rate of excretion of 3,5,6-trichloro-2-pyridinol derived from total void volume and results corrected for creatinine had been reported previously ${ }^{6}$ indicating that values corrected for creatinine are as valid for this purpose as rates of excretion based on volume. Nolan et $a l^{6}$ used doses $(0.5 \mathrm{mg} / \mathrm{kg}$ for the oral dose and $5 \mathrm{mg} / \mathrm{kg}$ for the dermal dose) which were sufficient to depress plasma cholinesterase values. The doses used in our study were insufficient to do this but were still detectable as dialkylphosphate metabolites in urine. This shows that the measurement of dialkylphosphate metabolites in urine is a more sensitive means of monitoring exposure to organophosphate pesticides.

The measurement of urinary dialkylphosphates is being increasingly used for biological monitoring of exposure to organophosphorus pesticides. The method can be used to monitor exposure to most of the organophosphorus pesticides in use. Previously exposure to chlorpyrifos had been measured mainly by determining 3,5,6-trichloro-2-pyridinol. We have established the apparent half life of chlorpyrifos dialkylphosphate metabolites for both an oral and dermal dose. If the exposure is through the dermal route the optimum sampling time is about 17-24 hours after the start of the shift during which exposure occurs. If exposure is mainly through inhalation or ingestion the optimum sampling time is 7 hours after dosing. An interesting finding is that after dermal exposure there is a prolonged period of elimination in the urine. This would mean that continued exposure to chlorpyrifos could lead to a cumulative concentration of dialkyl phosphate metabolites in the urine.

1 Sultanos LG, Shao M, Murphy SD. The role of hepatic biotransformation in mediating the acute toxicity of the phosphorothioate insecticide chlorpyrifos. Toxicol Appl Pharmacol 1984;73:60-8.

2 Richardson RJ. Assessment of the neurotoxic potential of chlorpyrifos relative to other organophosphorous compounds: a critical review of the literature. $\mathcal{F}$ Toxicol Environ Health 1995;44:135-65.

3 Fenske R, Elkner K. Multi route exposure assessment with biological monitoring of urban pesticide applicators during structural control treatments with chlorpyrifos. Toxicol Ind Health 1990;6:349-71.

4 Health and Safety Executive. Occupational exposure limits. London: The Stationary Office, 1998. (Publication: EH 40/98.)

5 Health and Safety Executive. Biological monitoring of workers exposed to organo-phosphorous pesticides. London: Her

6 Nolan RJ, Rick DL, Freshour NL, et al. Chlorpyrifos: pharmacokinetics in human volunteers. Toxicol Appl Pharmacol 1984;73:8-15.

7 Nutley BP, Cocker J. Biological monitoring of workers occupationally exposed to organophosphate pesticides. Pesticide Science 1993;38:315-22.

8 Aprea C, Sciarra G, Sartorelli P, et al. Biological monitoring of exposure to organophosphorous insecticides by assay of urinary alkylphosphates: influence of protective measures during manual operations with treated plants. Int Arch Occup Environ Health 1994;66:333-8.

9 Mason HJ, Lewis PJ. A study of the intra-individual variation in plasma and red cell cholinesterase activity and variation in plasma and red cell cholinesterase activity and pesticides. I Soc Occup Med 1989;39:121-4. 